\title{
铜镁铝三元水滑石的姜-泰勒效应
}

\author{
刘娇姚萍倪哲明* 李 远 施 炜
}

(浙江工业大学化学工程与材料学院, 先进催化材料实验室, 杭州 310032)

\begin{abstract}
摘要: 采用密度泛函理论(DFT), 用 CASTEP程序模块, 对类水滑石 $\left(\mathrm{Cu}_{x} \mathrm{Mg}_{3-x} \mathrm{Al}-\mathrm{LDHs}, x=0-3\right)$ 周期性模型进 行几何全优化, 通过分析各体系的结构参数、电子排布、氢键、Mulliken 电荷布居、结合能, 总结出体系中的姜-泰 勒效应和结构稳定性规律. 结果表明, 姜-泰勒效应不仅存在于 $d$ 轨道未排满的 $\mathrm{Cu}^{2+}$ 中, 在 $p$ 轨道未排满的 $\mathrm{Mg}^{2+}$ 中也可能存在, 且未饱和的 $d 、 p$ 轨道共同影响着金属离子姜-泰勒畸变的大小. 在 $\mathrm{Cu}_{x} \mathrm{Mg}_{3-x} \mathrm{Al}-\mathrm{LDHs}(x=0-3)$ 中, 铝八面体和镁八面体分别以稳定的拉长的八面体形式存在. 而随着 $\mathrm{Cu}^{2+}$ 的增加, 铜八面体逐渐从压扁的八面体 向稳定的拉长的八面体形式转变, 体系获得了逐渐增多的姜-泰勒稳定化能. 总体上, 随着 $\mathrm{Cu}^{2+}$ 的增加, 体系中 姜-泰勒效应导致的畸变使主客体间的氢键和静电作用力均有减弱趋势, 且体系的结合能绝对值逐渐减小, 故 体系稳定性下降. 这有助于从理论上进一步认识含铜水滑石的姜-泰勒效应.
\end{abstract}

关键词： 水滑石； 姜-泰勒效应；密度泛函理论； 结合能; Mulliken布居 中图分类号: 0641

\section{Jahn-Teller Effect of Cu-Mg-Al Layered Double Hydroxides}

\author{
LIU Jiao YAO Ping NI Zhe-Ming* LI Yuan SHI Wei \\ (Laboratory of Advanced Catalytic Materials, College of Chemical Engineering and Materials Science, \\ Zhejiang University of Technology, Hangzhou 310032, P. R. China)
}

\begin{abstract}
We propose a periodic interaction model for the layered double hydroxides, $\mathrm{Cu}_{x} \mathrm{Mg}_{3-\mathrm{x}} \mathrm{Al}-\mathrm{LDHs}$ $(x=0-3)$. Based on density functional theory, the geometries of $\mathrm{Cu}_{x} \mathrm{Mg}_{3-x} \mathrm{Al}-\mathrm{LDHs}(x=0-3)$ were optimized using the CASTEP program. The Jahn-Teller effect and the stability were investigated by analyzing the geometric parameters, electronic arrangement, hydrogen-bonding, charge populations, and binding energies. The results show that the Jahn-Teller effect exists in the unfilled $\mathrm{Cu}^{2+} d$ orbital and also exists in the unfilled $\mathrm{Mg}^{2+} p$ orbital. The two orbitals affect the Jahn-Teller distortion of the metal ions. In $\mathrm{Cu}_{x} \mathrm{Mg}_{3-x} \mathrm{Al}-$ $\operatorname{LDHs}(x=0-3)$, both aluminum and magnesium exist in stabilized octahedral forms. With an increase of $\mathrm{Cu}^{2+}$ in the layer the octahedral of copper changes from a flat configuration to a stable elongated configuration and the Jahn-Teller stabilization energy of the system gradually increases. In general, with an increase of $\mathrm{Cu}^{2+}$ in the layer the distortion caused by the Jahn-Teller effect weakens hydrogen-bonding and the electrostatic interactions between the host layer and the guest. The absolute value of the binding energy decreases and the chemical stability of the system decreases as well. This allows us to theoretically understand the Jahn-Teller effect better for the synthesis of copper-containing LDHs.
\end{abstract}

Key Words: Layered double hydroxide; Jahn-Teller effect; Density functional theory; Binding energy; Mulliken population

\footnotetext{
Received: May 9, 2011; Revised: July 8, 2011; Published on Web: July 15, 2011.
}

"Corresponding author. Email: jchx@zjut.edu.cn; Tel: +86-571-88320373

(C) Editorial office of Acta Physico-Chimica Sinica 


\section{1 引言}

层状双羟基阴离子粘土俗称水滑石(LDHs), 它 具有特殊的结构和性质. 铜是常用的催化活性组 元, 故含铜 LDHs 及其衍生物被广泛用在各种催化 反应中, ${ }^{1-3}$ 如合成安息香异丙醚反应、高氯酸铵的 热分解反应、 ${ }^{5}$ 丙炔催化加氢反应、 、,7 甲烷催化燃烧 反应 ${ }^{8}$ 等. 但作为独立的二价金属阳离子, $\mathrm{Cu}^{2+}$ 很难 进入 LDHs 层板内, 会形成扭曲的八面体结构而生 成 $\mathrm{Cu}(\mathrm{OH})_{2}$ 和 $\mathrm{CuO}$ 沉淀. ${ }^{9,10} \mathrm{Cu}^{2+}$ 不同于其它二价金属 阳离子的特殊性在于 $\mathrm{Cu}^{2+}$ 的姜-泰勒效应, 为从本质 上探究 $\mathrm{Cu}^{2+}$ 的姜-泰勒效应, 解释不同的铜离子配比 对 LDHs 微观结构及稳定性的影响, 有必要利用计 算机模拟技术.

密度泛函理论可以用来计算 LDHs 体系的结构 参数、成键状况、作用能、电子密度, 以及分子间交互 作用的电子性质 ${ }^{11-19}$ 等. Wei 等 ${ }^{12}$ 以簇模型 $\left[\mathrm{M}\left(\mathrm{OH}_{2}\right)_{6}\right]^{n+}$ $\left(\mathrm{M}\right.$ 为 $\mathrm{Li}^{+} 、 \mathrm{Mg}^{2+} 、 \mathrm{Cu}^{2+} 、 \mathrm{Al}^{3+} 、 \mathrm{Ga}^{3+}$ 等金属阳离子)为基 础, 采用混合密度泛函 B3LYP 方法 ${ }^{14,15}$ 讨论了体系 中姜-泰勒效应对结构参数的影响; 接着, 又采用密 度泛函理论-赝势平面波法 ${ }^{19}$ 对镁铝水滑石周期性 模型各体系的阳离子组成、排列方式以及簇模型的 堆积方式等进行了分析, 探究了体系的结构和性 质. 本课题组 ${ }^{20}$ 也曾用密度泛函理论对三元水滑石 $\left(\mathrm{Cu}_{x} \mathrm{Zn}_{3-x} \mathrm{Al}-\mathrm{LDHs}, x=0-3\right)$ 周期性模型各体系的结构 参数、氢键、Mulliken 电荷布居、结合能等进行分析, 研究了体系的畸变结构和稳定性.

基于以上基础, 本文采用密度泛函理论探究 $\mathrm{Cu}^{2+}$ 的姜-泰勒效应. 为缓解 $\mathrm{Cu}^{2+}$ 的姜-泰勒效应对层 板畸变的影响, 我们将 $\mathrm{Cu}^{2+}$ 掺杂进典型的镁铝水滑 石中, 从而形成三元含铜 $\mathrm{LDHs}\left(\mathrm{Cu}_{x} \mathrm{Mg}_{3-x} \mathrm{Al}-\mathrm{LDHs}\right.$, $x=1-3)$. 通过从理论上分析并解释体系的姜-泰勒效 应, 为进一步设计和制备多种具有姜-泰勒效应的金 属氧化物提供理论参考.

\section{2 计算模型和方法}

本文选取 $\mathrm{Mg}_{3} \mathrm{Al}(\mathrm{OH})^{8+}$ 为主体层板, $\mathrm{NO}_{3}^{-}$为客体 阴离子并处于 $\mathrm{Hcp}-\mathrm{Al}$ 位且平行地置于两层板的中 间(俯视时, $\mathrm{NO}_{3}^{-}$上的三个氧原子所形成的三角形处 于层板铝原子上的三个羟基氢所形成的三角形内 部), ${ }^{21,22}$ 以 $2 \mathrm{H}$ 堆积模式 ${ }^{23}$ 构建了镁铝水滑石 $\mathrm{Mg}_{6} \mathrm{Al}_{2}$ $(\mathrm{OH})_{16}\left(\mathrm{NO}_{3}\right)_{2}$ 模型, 如图 1(a, $\left.\mathrm{a}^{\prime}\right)$ 所示. 采用先前工作 中对水滑石体系较适用的计算方法: $:^{17,18,24}$ 选用 CASTEP 程序模块, ${ }^{25}$ 在 LDA-CA-PZ 基组水平 ${ }^{26}$ 对 模型进行几何全优化, 原子电子采用超软赝势 ${ }^{16}$ 进行 计算, 计算精度为 Medium, 自洽场计算的误差为 $2 \times$ $10^{-6} \mathrm{eV} \cdot \mathrm{atom}^{-1}$, 能带结构在布里渊区 $\boldsymbol{k}$ 矢量的选取 为 $4 \times 4 \times 1$, 截止能量为 $330.0 \mathrm{eV}$, 基态能量选用 Pulay 密度混合算法, ${ }^{27}$ 整体电荷数为 0 , 同时优化晶胞, 优 化后的微观结构及氢键作用模型如图 2(a)所示. 在 此基础上, 逐步用 $\mathrm{Cu}^{2+}$ 取代层板上的 $\mathrm{Mg}^{2+}$, 共构建了 5 个不同摩尔比或排布方式的 $\mathrm{Cu}_{x} \mathrm{Mg}_{3-x} \mathrm{Al}(\mathrm{OH})_{8} \mathrm{NO}_{3}$ (记作 $\mathrm{Cu}_{x} \mathrm{Mg}_{3-x} \mathrm{Al}-\mathrm{LDHs}(x=1-3)$ ), 模型如图 1(b, $\mathrm{b}^{\prime}$, $\mathrm{c}, \mathrm{c}^{\prime}, \mathrm{d}$ ) 所示. 通过对其进行几何全优化, 优化后(b) $\mathrm{CuMg}_{2} \mathrm{Al}-\mathrm{LDHs}-1$ 体系的能量 $\left(-1712399.8 \mathrm{~kJ} \cdot \mathrm{mol}^{-1}\right)$ 低于 ( $\left.b^{\prime}\right) \mathrm{CuMg}_{2} \mathrm{Al}-\mathrm{LDHs}-2$ 体系的能量(-1712398.1 $\mathrm{kJ} \cdot \mathrm{mol}^{-1}$ ), (c) $\mathrm{Cu}_{2} \mathrm{MgAl}-\mathrm{LDHs}-1$ 体系的能量 $\left(-1786570.2 \mathrm{~kJ} \cdot \mathrm{mol}^{-1}\right)$ 低于 $\left(\mathrm{c}^{\prime}\right) \mathrm{Cu}_{2} \mathrm{MgAl}-\mathrm{LDHs}-2$ 体 系的能量 $\left(-1786562.1 \mathrm{~kJ} \cdot \mathrm{mol}^{-1}\right)$, 说明在相同 $\mathrm{Cu}^{2+} /$ $\mathrm{Mg}^{2+}$ 摩尔比的情况下, 层板上 $\mathrm{Cu}^{2+}$ 与 $\mathrm{Mg}^{2+}$ 交错排列 的构型比较稳定. 故我们选择其中最稳定的四种不

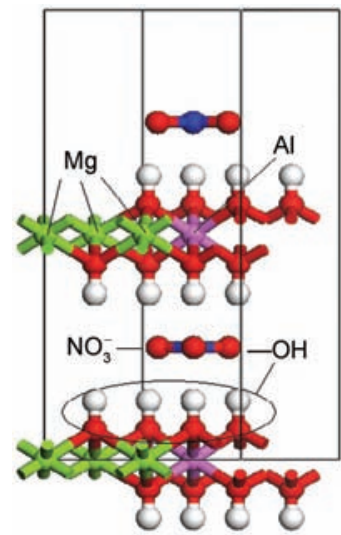

(a') $\mathrm{Mg}_{3} \mathrm{Al}$-LDHs

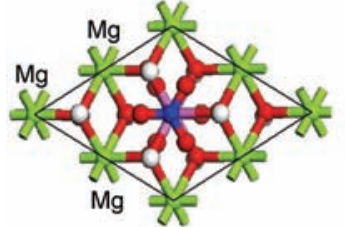

(a) $\mathrm{Mg}_{3} \mathrm{Al}-\mathrm{LDHs}$

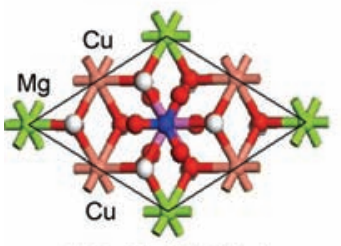

(c) $\mathrm{Cu}_{2} \mathrm{MgAl}-\mathrm{LDHs}-1$

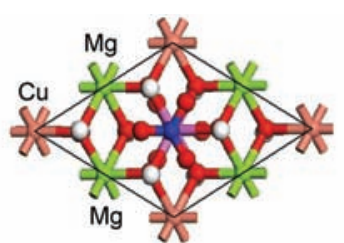

(b) CuMg_Al-LDHs-1

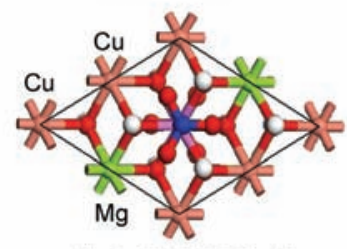

(c') $\mathrm{Cu}_{2} \mathrm{MgAl}-\mathrm{LDHs}-2$

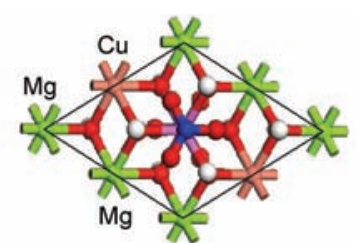

(b') $\mathrm{CuMg}_{2} \mathrm{Al}-\mathrm{LDHs}-2$

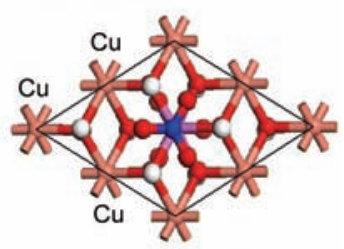

(d) $\mathrm{Cu}_{3} \mathrm{Al}-\mathrm{LDHs}$

图 $1 \mathrm{Cu}_{x} \mathrm{Mg}_{3-x} \mathrm{Al}-\mathrm{LDHs}(x=0-3)$ 主客体计算模型

Fig.1 Host-guest calculation models of $\mathrm{Cu}_{x} \mathrm{Mg}_{3-x} \mathrm{Al}-\mathrm{LDHs}(x=0-3)$ 

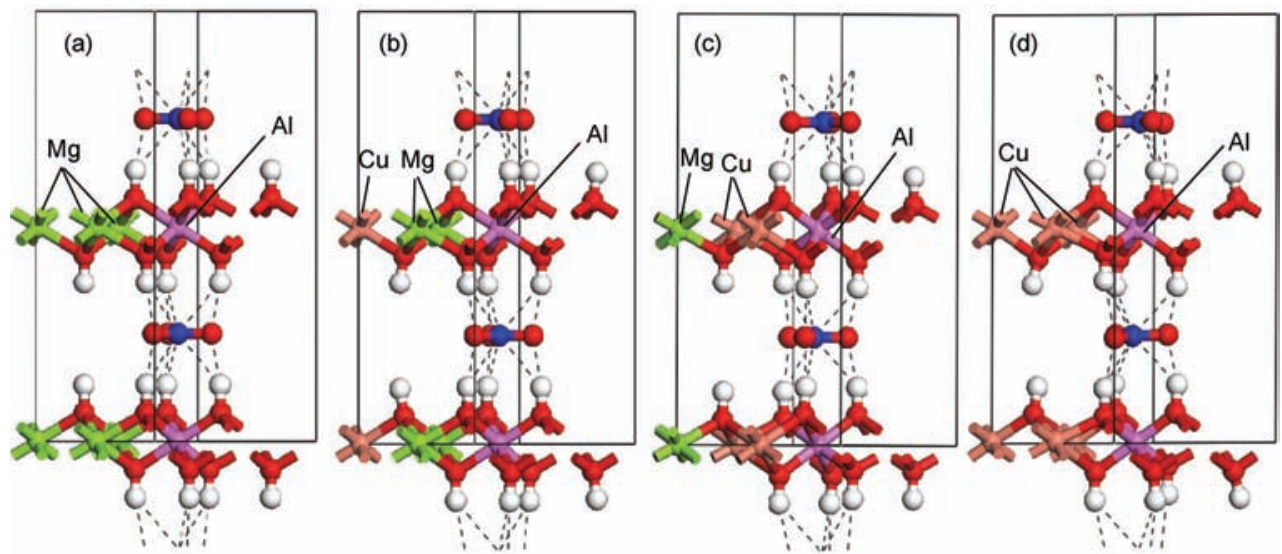

$\mathrm{Mg}_{3} \mathrm{Al}-\mathrm{LDHs}$ (system 1) $\quad \mathrm{CuMg}_{2} \mathrm{Al}-\mathrm{LDH}$ (system 2) $\quad \mathrm{Cu}_{2} \mathrm{MgAl}-\mathrm{LDHs}$ (system 3) $\mathrm{Cu}_{3} \mathrm{Al}-\mathrm{LDHs}$ (system 4)

图 $2 \mathrm{Cu}_{x} \mathrm{Mg}_{3-x} \mathrm{Al}-\mathrm{LDHs}(x=0-3)$ 优化构型和层间氢键分布

Fig.2 Optimized geometrical structures and the distribution of hydrogen-bonding between layers of

\section{$\mathrm{Cu}_{x} \mathrm{Mg}_{3-x} \mathrm{Al}-\operatorname{LDHs}(\boldsymbol{x}=\mathbf{0 - 3})$}

Short dashed line denotes the hydrogen-bonding between host and guest.

同 $\mathrm{Cu}^{2+} / \mathrm{Mg}^{2+}$ 摩尔比体系如图 1(a, b, c, d) 用于计算, 将优化后的(a)记作 1 号体系 $\left(\mathrm{Mg}_{3} \mathrm{Al}-\mathrm{LDHs}\right)$, (b) 记作 2 号体系 $\left(\mathrm{CuMg}_{2} \mathrm{Al}-\mathrm{LDHs}\right)$, (c) 记作 3 号体系 ( $\left.\mathrm{Cu}_{2} \mathrm{MgAl}-\mathrm{LDHs}\right)$, (d) 记作 4 号体系( $\left.\mathrm{Cu}_{3} \mathrm{Al}-\mathrm{LDHs}\right)$.

\section{3 结果与讨论}

\section{1 结构参数分析}

表 1 为 $\mathrm{Cu}_{x} \mathrm{Mg}_{3-x} \mathrm{Al}-\mathrm{LDHs}(x=0-3)$ 体系优化后的 结构参数. 晶胞参数 $c$ 值 (层间距 $d_{c}=0.5 c$ ) 为层板厚 度和层间通道大小的加和, 故其值不仅受到层板中 金属离子半径的影响, 还受到主客体间作用力大小 的影响. ${ }^{28}$ 表 2 中列出了 $\mathrm{Cu}_{x} \mathrm{Mg}_{3-x} \mathrm{Al}-\mathrm{LDHs}(x=0-3)$ 体 系优化后主要键长的平均值. 由表 2 可见, 1 号体系
的最长键是 $l_{\mathrm{Mg}-\mathrm{O}}, 2-4$ 号体系的最长键是 $l_{\mathrm{Cu}-\mathrm{O}}$, 故其 在决定体系层板厚度中起一定作用. 从 1 号到 4 号 体系, 它们最长键的键长逐渐增加, 1-3 号体系的 $c$ 值逐渐上升, 到 4 号体系时却明显下降. 这可能是由 于在 $\mathrm{Cu}_{x} \mathrm{Mg}_{3-x} \mathrm{Al}-\mathrm{LDHs}$ 体系中镁的电荷布居为 $1.680 e-2.010 e$, 铜的电荷布居为 $0.520 e-0.863 e$, 即 镁的电荷布居大于铜的电荷布居, 故从 1 号到 3 号 体系, 随着铜取代镁的增加, 层板电荷密度随之减 小, 削弱了层板正电荷与层间阴离子间的静电引 力, 使层间通道增大, $c$ 值增加; 而在 4 号体系中随着 LDHs 体系中 $\mathrm{Cu}^{2+}$ 掺杂的增多, 体系的畸变程度变 大, 为缓解这种不稳定性, 主客体相互靠近以获得 更多的主客体作用力, 从而维持体系的相对稳定性.

表 $1 \mathrm{Cu}_{x} \mathrm{Mg}_{3-x} \mathrm{Al}-\mathrm{LDHs}(x=0-3)$ 的晶胞参数

Table 1 Lattice parameters of $\mathrm{Cu}_{x} \mathrm{Mg}_{3-x} \mathrm{Al}-\operatorname{LDHs}(x=0-3)$

\begin{tabular}{|c|c|c|c|c|c|c|c|}
\hline \multirow{2}{*}{ System } & \multicolumn{3}{|c|}{ Lattice length/nm } & \multicolumn{4}{|c|}{ Lattice angle $/\left({ }^{\circ}\right)$} \\
\hline & $a$ & $b$ & $c$ & $\alpha$ & $\beta$ & $\gamma$ & $\Delta \theta_{\alpha+\beta+y}$ \\
\hline $\mathrm{Mg}_{3} \mathrm{Al}-\mathrm{LDHs}$ (system 1) & 0.61301 & 0.61284 & 1.51414 & 90.001 & 89.993 & 119.948 & 0.060 \\
\hline $\mathrm{CuMg}_{2} \mathrm{Al}-\mathrm{LDHs}$ (system 2) & 0.60755 & 0.60610 & 1.51721 & 89.997 & 90.010 & 118.182 & 1.831 \\
\hline $\mathrm{Cu}_{2} \mathrm{MgAl}-\mathrm{LDHs}$ (system 3) & 0.62136 & 0.62064 & 1.52343 & 90.017 & 89.992 & 121.923 & 1.207 \\
\hline $\mathrm{Cu}_{3} \mathrm{Al}-\mathrm{LDHs}$ (system 4) & 0.63762 & 0.58015 & 1.50299 & 90.400 & 89.585 & 117.211 & 3.604 \\
\hline
\end{tabular}

$\Delta \theta_{\alpha+\beta+\gamma}$ : the deviation value between the sum of lattice angles $\alpha, \beta, \gamma$ and the ideal lattice angles $\left(\alpha=90^{\circ}, \beta=90^{\circ}, \gamma=120^{\circ}\right)$

\section{表 $2 \mathrm{Cu}_{x} \mathrm{Mg}_{3-x} \mathrm{Al}-\mathrm{LDHs}(x=0-3)$ 的优化键长}

Table 2 Optimized bond length of $\mathrm{Cu}_{x} \mathrm{Mg}_{3-x} \mathrm{Al}-\mathrm{LDHs}(x=0-3)$

\begin{tabular}{lccccccc}
\hline \multicolumn{1}{c}{ System } & $l_{\mathrm{Al}-\mathrm{O}} / \mathrm{nm}$ & $l_{\mathrm{Mg}-\mathrm{O}} / \mathrm{nm}$ & $l_{\mathrm{Cu}-\mathrm{O}(\mathrm{S})} / \mathrm{nm}$ & $l_{\mathrm{Cu}-\mathrm{OL}(\mathrm{L})} / \mathrm{nm}$ & $l_{\mathrm{Cu}-\mathrm{O}} / \mathrm{nm}$ & $l_{\mathrm{O}-\mathrm{H}} / \mathrm{nm}$ & $l_{\mathrm{N}-\mathrm{o}} / \mathrm{nm}$ \\
\hline $\mathrm{Mg}_{3} \mathrm{Al}-\mathrm{LDHs}$ (system 1) & 0.19030 & 0.20755 & - & - & - & 0.09866 & 0.12697 \\
$\mathrm{CuMg}_{2} \mathrm{Al}-L D H s$ (system 2) & 0.18931 & 0.20721 & 0.18992 & 0.21707 & 0.20802 & 0.09876 & 0.12697 \\
$\mathrm{Cu}_{2} \mathrm{MgAl}-L D H s$ (system 3) & 0.18951 & 0.20684 & 0.19618 & 0.23261 & 0.20833 & 0.09895 & 0.12699 \\
$\mathrm{Cu}_{3} \mathrm{Al}-\mathrm{LDHs}$ (system 4) & 0.18927 & - & 0.19360 & 0.24337 & 0.21019 & 0.09921 & 0.12705 \\
\hline
\end{tabular}


表 $3 \mathrm{Cu}_{x} \mathrm{Mg}_{3-x} \mathrm{Al}-\mathrm{LDHs}(x=0-3)$ 的 $\mathrm{Cu}-\mathrm{O}$ 键长 Table $3 \mathrm{Cu}-\mathrm{O}$ bond length of $\mathrm{Cu}_{x} \mathrm{Mg}_{3-x} \mathrm{Al}-\mathrm{LDHs}(x=0-3)$

\begin{tabular}{lcccccc}
\hline \multicolumn{1}{c}{ System } & $l_{\mathrm{Cu}-\mathrm{O} 1} / \mathrm{nm}$ & $l_{\mathrm{Cu}-\mathrm{O} 2} / \mathrm{nm}$ & $l_{\mathrm{Cu}-\mathrm{O} 3} / \mathrm{nm}$ & $l_{\mathrm{Cu}-\mathrm{O} /} / \mathrm{nm}$ & $l_{\mathrm{Cu}-\mathrm{O} 5} / \mathrm{nm}$ & $l_{\mathrm{Cu}-\mathrm{O} \sigma} / \mathrm{nm}$ \\
\hline $\mathrm{CuMg}_{2} \mathrm{Al}-\mathrm{LDHs}$ (system 2) & 0.18989 & 0.18995 & 0.21517 & 0.21555 & 0.21871 & 0.21885 \\
$\mathrm{Cu}_{2} \mathrm{MgAl}-L D H s$ (system 3) & 0.19206 & 0.19215 & 0.19945 & 0.20082 & 0.23211 & 0.23301 \\
$\mathrm{Cu}_{3} \mathrm{Al}$-LDHs (system 4) & 0.18828 & 0.19190 & 0.19458 & 0.19964 & 0.23975 & 0.24698 \\
\hline
\end{tabular}

晶胞参数 $a$ 和 $b$ 值的大小主要归因于不同层板 金属离子半径对其的影响, ${ }^{28}$ 即在 $\mathrm{Cu}_{x} \mathrm{Mg}_{3-x} \mathrm{Al}-\mathrm{LDHs}$ 体系中, 主要是与晶胞棱上的 $\mathrm{Al}-\mathrm{O} / \mathrm{Mg}-\mathrm{O} / \mathrm{Cu}-\mathrm{O}$ 键长大小相关.

从晶胞角度 $\alpha 、 \beta 、 \gamma$ 与理想六方晶胞角度 $\left(\alpha=90^{\circ}\right.$, $\left.\beta=90^{\circ}, \gamma=120^{\circ}\right)$ 的偏离值之和 $\Delta \theta_{\alpha+\beta+\gamma}$ 中可以看出, 1 号、 2 号、 3 号、 4 号体系的晶胞畸变程度有变大趋势. 1 号体系并无掺杂 $\mathrm{Cu}^{2+}$, 故其晶型最好; 2 号体系的 晶胞畸变程度反而大于 3 号体系, 这是因为 2 号体 系的 $\mathrm{Cu}^{2+}$ 排在晶胞顶点位置, 其畸变直接影响晶胞 的畸变情况; 4 号体系的二价阳离子全部是 $\mathrm{Cu}^{2+}$, 姜泰勒效应最显著, 故其畸变最大.

\section{2 姜-泰勒效应分析}

1937 年, 姜(Jahn, H. A.)和泰勒(Teller, E.)指出: 在对称的非线性分子中, 如果一个体系的基态有几 个简并能级, 则是不稳定的, 体系一定要发生畸变, 使一个能级降低, 以消除简并性, 这就是姜-泰勒效 应. ${ }^{29}$ 若失去的是 $d_{z^{2}}$ 上的电子, 则会变成压扁的八 面体构型; 若失去的是 $d_{x^{2}-y^{2}}$ 上的电子, 则会变成拉 长的八面体构型. ${ }^{29}$

表 3 列出了 $\mathrm{Cu}_{x} \mathrm{Mg}_{3-x} \mathrm{Al}-\mathrm{LDHs}$ 体系中铜配位体 的键长(以上层板为例). 从表中可看出, 2 号体系中 的六个 $\mathrm{Cu}-\mathrm{O}$ 键出现了两短键四长键的分化, 为典 型的压扁的八面体; 3 号体系中的 $\mathrm{Cu}-\mathrm{O}$ 键出现了 四短键两长键的分化形式, 相比 2 号体系, 它的其中 两个长键变短, 而另两个长键变长; 4 号体系中的六 个 $\mathrm{Cu}-\mathrm{O}$ 键, 相比 3 号体系, 它的四个短键变得更 短, 两个长键变得更长, 最终出现了四短键两长键 的分化, 为典型的拉长的八面体. 实验证明, 铜的六 配位配合物以拉长的八面体形式稳定存在, 这是因
为在无其它能量因素影响时, 形成两条长键四条短 键比形成两条短键四条长键的总键能要大. ${ }^{29}$ 故从 2 号到 4 号体系, 铜八面体配合物获得了逐渐增多的 姜-泰勒稳定化能, 有利于缓解畸变导致的不稳定性 的影响. 另外在铝配位体中, 1 号体系中各个 $\mathrm{Al}-\mathrm{O}$ 键长值相差不大, 从 2 号到 4 号体系, $\mathrm{Al}-\mathrm{O}$ 键长出 现了长短之分, 为四个共面的短键和两个与该面垂 直的长键. 总体上, 从 1 号到 4 号体系, $\mathrm{Al}-\mathrm{O}$ 长键依 次增加 $(0.19046,0.19062,0.19786,0.19856 \mathrm{~nm})$, 其 短键依次减小 $(0.19023,0.18866,0.18534,0.18463$ $\mathrm{nm})$, 故在 $\mathrm{Cu}_{x} \mathrm{Mg}_{3-x} \mathrm{Al}-\mathrm{LDHs}(x=1-3)$ 体系中, 铝的六 配位配合物逐渐向拉长的八面体形式转化并稳定 存在于层板中. 在镁配位体中, 1 号体系的 $\mathrm{Mg}-\mathrm{O}$ 键长先出现长短之分, 为四个共面的短键和两个与 该面垂直的长键, 即在典型的镁铝水滑石中, 镁的 六配位配合物是以拉长的八面体形式存在的. 从 1 号到 3 号体系, $\mathrm{Mg}-\mathrm{O}$ 键长没有明显变化, 铜的加 入对其影响不大.

对于金属离子的角度畸变程度, 可用 $\Delta \theta_{\text {омо }}(\mathrm{M}$ 为 $\mathrm{Cu} 、 \mathrm{Mg} 、 \mathrm{Al})$ 来衡量, 即层板中每一个金属原子与其相 配位的氧原子所形成的共 12 个角 $\theta_{\text {омо }}$ 跟理想六配位 角度 $\left(90^{\circ}\right)$ 的绝对差值的平均值. $\mathrm{Cu}_{x} \mathrm{Mg}_{3-x} \mathrm{Al}-\mathrm{LDHs}$ 体 系中的 $\mathrm{Cu}^{2+}, 3 d$ 轨道电子为 $9.423 e-9.430 e$, 为不饱 和的 $10 e$ 状态, 故产生了姜-泰勒畸变. 由表 4 可见, 从 2 号到 4 号体系, $\Delta \theta_{\mathrm{OCu}}$ 逐渐变大, 是由于 $\mathrm{Cu}^{2+}$ 的 $3 d$ 轨道电子依次减小, 逐渐远离饱和的 $d^{10}$ 状态, 故所获得的姜-泰勒稳定化能逐渐增多. 另外, 在 $\mathrm{Cu}_{x} \mathrm{Mg}_{3-x} \mathrm{Al}-\mathrm{LDHs}$ 体系中, 无 $d$ 轨道电子的镁和铝配 合物也有一定程度的角度畸变, 且 $\Delta \theta_{\mathrm{ongo}}$ 值仅略低 于 $\Delta \theta_{\mathrm{OCuo}}$ 值 ( 2 号体系中 $\Delta \theta_{\mathrm{OMgO}}$ 值略高于 $\Delta \theta_{\mathrm{OCuo}}$ 值). 据

表 $4 \mathrm{Cu}_{x} \mathrm{Mg}_{3-x} \mathrm{Al}-\mathrm{LDHs}(x=0-3)$ 体系中的金属畸变角及原子的轨道布居

Table 4 Distortion angle and atomic orbital populations of $\mathrm{Cu}_{x} \mathrm{Mg}_{3-x} \mathrm{Al}-\mathrm{LDHs}(x=0-3)$

\begin{tabular}{|c|c|c|c|c|c|c|c|c|}
\hline \multirow{2}{*}{ System } & \multirow{2}{*}{$\Delta \theta_{\mathrm{OAIO}} /\left(^{\circ}\right)$} & \multirow{2}{*}{$\Delta \theta_{\mathrm{OMgO}} /\left(^{\circ}\right)$} & \multirow{2}{*}{$\Delta \theta_{\mathrm{OCuO}} /\left(^{\circ}\right)$} & \multicolumn{4}{|c|}{ Orbital population/e } & \multirow{2}{*}{$\Delta \theta_{\mathrm{OMO}} /\left(^{\circ}\right)$} \\
\hline & & & & Al $3 p$ & $\operatorname{Mg} 2 p$ & $\mathrm{Cu} 4 p$ & $\mathrm{Cu} 3 d$ & \\
\hline $\mathrm{Mg}_{3} \mathrm{Al}-\mathrm{LDHs}$ (system 1) & 5.337 & 8.850 & - & 1.03 & 5.91 & - & - & 7.972 \\
\hline $\mathrm{CuMg}_{2} \mathrm{Al}-\mathrm{LDHs}$ (system 2) & 4.719 & 9.141 & 9.086 & 0.98 & 5.79 & 0.50 & 9.430 & 8.022 \\
\hline $\mathrm{Cu}_{2} \mathrm{MgAl}-\mathrm{LDHs}$ (system 3) & 4.421 & 9.926 & 10.745 & 0.91 & 5.68 & 0.37 & 9.425 & 8.889 \\
\hline $\mathrm{Cu}_{3} \mathrm{Al}$-LDHs (system 4) & 4.889 & - & 10.971 & 0.83 & - & 0.25 & 9.423 & 9.451 \\
\hline
\end{tabular}

$\Delta \theta_{\text {омо: }}$ the system's average metal distortion angle 
此推测, 不仅只有 $d$ 轨道电子的不均匀排布会发生 姜-泰勒畸变, $p$ 轨道电子的不均匀排布也可能会发 生姜-泰勒畸变. 从理论上来说, $p_{x} 、 p_{y} 、 p_{z}$ 中任一轨道 上的电子缺失或者多余, 导致三个轨道的电子排布 不相同, 相应轴上的 $\mathrm{M}-\mathrm{O}$ 键就会由于斥力的改变 而缩短或者拉长, 从而导致畸变的产生.

故考虑体系中金属离子 $d$ 轨道的不均匀排布、 $p$ 轨道的不均匀排布及受周围配合物畸变的影响三 方面因素, $\mathrm{Cu}_{x} \mathrm{Mg}_{3-x} \mathrm{Al}-\mathrm{LDHs}$ 体系中金属配合物的 畸变程度大小顺序为 $\Delta \theta_{\mathrm{OCuO}}>\Delta \theta_{\mathrm{OMgO}}>\Delta \theta_{\mathrm{OAlO}}$; 且从 1 号

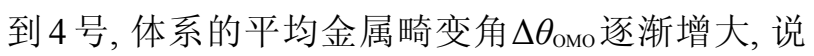
明体系获得了逐渐增多的姜-泰勒稳定化能, 有利于 体系的稳定性. 但姜-泰勒稳定化能仅能部分缓解由 其畸变带来的结构不稳定的影响, ${ }^{20}$ 故最终导致体 系的稳定性下降.

\section{3 作用力分析}

\subsection{1 氢键网络分析}

氢键是氢原子与电负性的原子 $\mathrm{X}$ 共价结合时, 共用的电子对强烈地偏向 $\mathrm{X}$ 的一边, 使氢原子带有 部分正电荷, 能再与另一个电负性高而半径较小的 原子 $\mathrm{Y}$ 结合, 形成的 $\mathrm{X}-\mathrm{H} \cdots \mathrm{Y}$ 型的键. 不仅同种分 子之间可以存在氢键, 某些不同种分子之间也可能 形成氢键. 一般情况下氢键具有饱和性和方向性, 且氢键的键长越短, 键角越接近于 $180^{\circ}$, 氢键的强 度越强.

在 $\mathrm{Cu}_{x} \mathrm{Mg}_{3-x} \mathrm{Al}-\mathrm{LDHs}$ 体系中层间阴离子电负性 较强的原子并不只与层板上的一个羟基中的氢原 子形成氢键, 而是与多个羟基上的氢原子形成多重 氢键; 单个羟基氢原子还可与多个电负性原子形成 多重氢键, 故 $\mathrm{Cu}_{x} \mathrm{Mg}_{3-x} \mathrm{Al}-\mathrm{LDHs}$ 体系中存在复杂的 氢键网络. 总体上, $\mathrm{Cu}_{x} \mathrm{Mg}_{3-x} \mathrm{Al}-\mathrm{LDHs}$ 体系中的 $\mathrm{NO}_{3}^{-}$ 与层板形成两类氢键: (1) $\mathrm{NO}_{3}^{-}$上的氧原子与层板上 的 $-\mathrm{OH}$ 形成的氢键, 记作 $\mathrm{O}-\mathrm{H} \cdots \mathrm{O}$; (2) $\mathrm{NO}_{3}^{-}$上的 氮原子与层板上的 $-\mathrm{OH}$ 形成的氢键, 记作 $\mathrm{O}-\mathrm{H} \cdots$ $\mathrm{N}$. 由图 3 可知, $\mathrm{O}-\mathrm{H} \cdots \mathrm{O}$ 型氢键比 $\mathrm{O}-\mathrm{H} \cdots \mathrm{N}$ 型氢 键的键角更接近 $180^{\circ}$, 键长更短, 故其氢键强度更
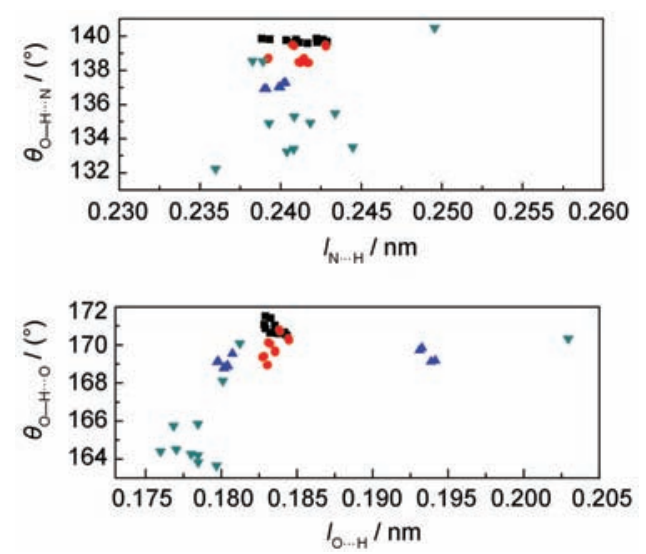

图 $3 \mathrm{Cu}_{x} \mathrm{Mg}_{3-x} \mathrm{Al}-\mathrm{LDHs}(x=0-3)$ 中氢键的键长和键角的 关系

Fig.3 Relationship between bond angle and bond length of hydrogen-bond contained in $\mathrm{Cu}_{x} \mathrm{Mg}_{3-x} \mathrm{Al}-\mathrm{LDHs}(x=\mathbf{0 - 3})$ - $\mathrm{Mg}_{3} \mathrm{Al}$-LDHs, $\mathrm{Mg}_{2} \mathrm{CuAl}$-LDHs, $\triangle \mathrm{MgCu}_{2} \mathrm{Al}$-LDHs, $\checkmark \mathrm{Cu}_{3} \mathrm{Al}-\mathrm{LDHs}$

大. 此外, 随着 $\mathrm{Cu}^{2+}$ 取代的增加, 两种类型的氢键逐 渐分散, 是由于从 1 号到 4 号体系, 金属的平均畸变 角逐渐变大, 层板的金属畸变大小影响氢键分散程 度, 即金属畸变越严重, 氢键越分散.

从 1 号到 4 号体系, $\mathrm{O}-\mathrm{H} \cdots \mathrm{O}$ 型氢键的 $\mathrm{H} \cdots \mathrm{O}$ 键的平均键长分别为 $0.18350,0.18348,0.18518$, $0.18282 \mathrm{~nm}, \mathrm{O}-\mathrm{H} \cdots \mathrm{N}$ 型氢键的 $\mathrm{H} \cdots \mathrm{N}$ 键的平均键 长分别为 $0.24129,0.24118,0.23544,0.24124 \mathrm{~nm}$, 两 种类型的氢键键长变化并不明显. 但由图 3 可看出, 这两类氢键均逐渐向图的下端偏移, 即氢键键角明 显逐渐远离 $180^{\circ}$. 故结合键长和键角的分析可知, 随着 $\mathrm{Cu}^{2+}$ 取代的增加, 氢键强度总体上逐渐减弱.

\subsubsection{Mulliken 电荷布居分析}

Mulliken 布居是 Mulliken ${ }^{30}$ 提出的表示电荷在 各组成原子之间分布情况的方法. 为了进一步分析 $\mathrm{Cu}_{x} \mathrm{Mg}_{3-x} \mathrm{Al}-\mathrm{LDHs}$ 体系的作用力, 表 5 和表 6 列出了 体系的 Mulliken 电荷布居(对上下的层板、客体阴离 子、各原子及键的电荷布居作了平均处理).

$\mathrm{Cu}_{x} \mathrm{Mg}_{3-x} \mathrm{Al}-\mathrm{LDHs}$ 体系中铝的电荷布居为 $1.370 e-1.660 e$, 镁的电荷布居为 $1.680 e-2.010 e$, 铜的

表 $5 \mathrm{Cu}_{x} \mathrm{Mg}_{3-x} \mathrm{Al}-\operatorname{LDHs}(x=0-3)$ 的 Mulliken 电荷布居

Table 5 Mulliken atomic population of $\mathrm{Cu}_{x} \mathrm{Mg}_{3-x} \mathrm{Al}-\mathrm{LDHs}(x=0-3)$

\begin{tabular}{lccccccccc}
\hline \multirow{2}{*}{ System } & \multicolumn{7}{c}{ Charge population $/ e$} \\
\cline { 2 - 11 } & $\mathrm{Al}$ & $\mathrm{Mg}$ & $\mathrm{Cu}$ & $\mathrm{N}$ & $\mathrm{O}\left(\mathrm{NO}_{3}^{-}\right)$ & $\mathrm{O}(-\mathrm{OH})$ & $\mathrm{H}$ & $\mathrm{NO}_{3}^{-}$ & layer \\
\hline $\mathrm{Mg}_{3} \mathrm{Al}-\mathrm{LDHs}$ (system 1) & 1.370 & 1.680 & - & 0.550 & -0.430 & -1.103 & 0.400 & -0.740 & 0.785 \\
$\mathrm{CuMg}_{2} \mathrm{Al}-\mathrm{LDHs}$ (system 2) & 1.450 & 1.855 & 0.520 & 0.560 & -0.433 & -1.020 & 0.402 & -0.740 & 0.755 \\
$\mathrm{Cu}_{2} \mathrm{MgAl}-\mathrm{LDHs}$ (system 3) & 1.550 & 2.010 & 0.705 & 0.560 & -0.433 & -0.939 & 0.413 & -0.740 & 0.750 \\
$\mathrm{Cu}_{3} \mathrm{Al}-\mathrm{LDHs}$ (system 4) & 1.660 & - & 0.863 & 0.560 & -0.433 & -0.854 & 0.417 & -0.740 & 0.750 \\
\hline
\end{tabular}


表 $6 \mathrm{Cu}_{x} \mathrm{Mg}_{3-x} \mathrm{Al}-\mathrm{LDHs}(x=0-3)$ 的 Mulliken 成键布居 $(e)$

Table 6 Mulliken bond population of $\mathrm{Cu}_{x} \mathrm{Mg}_{3-x} \mathrm{Al}-\mathrm{LDHs}(x=0-3)$

\begin{tabular}{lccccccc}
\hline \multirow{2}{*}{ System } & \multicolumn{7}{c}{ Charge population/e } \\
\cline { 2 - 8 } & $\mathrm{N}-\mathrm{O}$ & $\mathrm{H}-\mathrm{O}$ & $\mathrm{Al}-\mathrm{O}$ & $\mathrm{Mg}-\mathrm{O}$ & $\mathrm{Cu}-\mathrm{O}$ & $\mathrm{H} \cdots \mathrm{O}$ & $\mathrm{H} \cdots \mathrm{N}$ \\
\hline $\mathrm{Mg}_{3} \mathrm{Al}-\mathrm{LDHs}$ (system 1) & 0.680 & 0.572 & 0.420 & -0.848 & - & 0.055 & -0.050 \\
$\mathrm{CuMg}_{2} \mathrm{Al}-\mathrm{LDHs}$ (system 2) & 0.682 & 0.578 & 0.400 & -0.913 & 0.263 & 0.052 & -0.050 \\
$\mathrm{Cu}_{2} \mathrm{MgAl}-\mathrm{LDHs}$ (system 3) & 0.680 & 0.579 & 0.367 & -0.982 & 0.210 & 0.057 & -0.052 \\
$\mathrm{Cu}_{3} \mathrm{Al}$-LDHs (system 4) & 0.680 & 0.590 & 0.338 & - & 0.174 & 0.058 & -0.049 \\
\hline
\end{tabular}

电荷布居为 $0.520 e-0.863 e$, 说明在该体系中, 金属 阳离子与其它阴离子的静电作用力大小顺序为: $\mathrm{Mg}^{2+}>\mathrm{Al}^{3+}>\mathrm{Cu}^{2+} ; \mathrm{Al}-\mathrm{O}$ 间的重叠布居数为 $0.338 e-$ $0.420 e, \mathrm{Mg}-\mathrm{O}$ 间重叠布居数为 $-0.982 e--0.848 e$, $\mathrm{Cu}-\mathrm{O}$ 间的重叠布居数为 $0.174 e-0.263 e$, 说明键的 强弱顺序为: $\mathrm{Mg}-\mathrm{O}>\mathrm{Al}-\mathrm{O}>\mathrm{Cu}-\mathrm{O}$. 据此可以解 释, 天然矿物中存在水镁石和典型的镁铝水滑石, 而含锌水滑石则需人工合成, 含铜水滑石的稳定性 较差. ${ }^{9,1023}$ 故在 $\mathrm{Cu}_{x} \mathrm{Mg}_{3-x} \mathrm{Al}-\mathrm{LDHs}$ 体系中, $\mathrm{Cu}^{2+}$ 掺杂的 越多, 体系的稳定性越差.

在 $\mathrm{Cu}_{x} \mathrm{Mg}_{3-x} \mathrm{Al}-\mathrm{LDHs}(x=0-3)$ 体系层间引入阴 离子 $\mathrm{NO}_{3}^{-}$后, 层板的电荷由 $1.00 e$ 分别降为 $0.785 e$, $0.755 e, 0.750 e, 0.750 e$, 说明电子由层间客体阴离子 向主体层板发生了部分转移. 总体上, 从 1 号到 4 号 体系, 客体 $\mathrm{NO}_{3}^{-}$电荷均为 $-0.740 e$, 而层板电荷有减 小趋势, 故主客体间的静电作用力减弱.

\section{3 .3 结合能分析}

$\mathrm{Cu}_{x} \mathrm{Mg}_{3-x} \mathrm{Al}-\mathrm{LDHs}$ 体系(包含客体阴离子 $\mathrm{NO}_{3}^{-}$) 的结合能定义式如下:

$$
\begin{aligned}
& \Delta E_{\mathrm{Cu}_{x} \mathrm{Mg}_{3-x} \mathrm{Al}-\mathrm{LDHs}}=E_{\mathrm{Cu}_{x} \mathrm{Mg}_{3-x} \mathrm{Al}-\mathrm{LDHs}}- \\
&\left(16 E_{\mathrm{H}}+2 E_{\mathrm{N}}+22 E_{\mathrm{O}}+2 E_{\mathrm{Al}}+n_{1} E_{\mathrm{Cu}}+n_{2} E_{\mathrm{Mg}}\right)
\end{aligned}
$$

其中, $\Delta E_{\mathrm{Cu}_{x} \mathrm{Mg}_{3-x} \mathrm{Al}-\mathrm{LDHs}}$ 表示体系总的结合能, 反映 了计算的体系的相对稳定性, $E_{\mathrm{Cu}_{x} \mathrm{Mg}_{3-x} \mathrm{Al}-\mathrm{LDHs}}$ 为 $\mathrm{Cu}_{x} \mathrm{Mg}_{3-x} \mathrm{Al}-\mathrm{LDHs}$ 体系的能量, $E_{\mathrm{H}}=-1174.4 \mathrm{~kJ}$. $\mathrm{mol}^{-1}, E_{\mathrm{N}}=-25288.9 \mathrm{~kJ} \cdot \mathrm{mol}^{-1}, E_{0}=-41376.3 \mathrm{~kJ} \cdot$ $\mathrm{mol}^{-1}, E_{\mathrm{Al}}=-5515.5 \mathrm{~kJ} \cdot \mathrm{mol}^{-1}, E_{\mathrm{Cu}}=-130219.3 \mathrm{~kJ} \cdot$ $\mathrm{mol}^{-1}, E_{\mathrm{Mg}}=-93793.7 \mathrm{~kJ} \cdot \mathrm{mol}^{-1}$ 为各原子的能量, $n_{1}$ 为 单个晶胞中铜原子的数目, $n_{2}$ 为单个晶胞中镁原子

表 $7 \mathrm{Cu}_{x} \mathrm{Mg}_{3-x} \mathrm{Al}-\operatorname{LDHs}(x=0-3)$ 的结合能

\begin{tabular}{|c|c|c|}
\hline System & $\frac{E_{\mathrm{Cu}_{x} \mathrm{Mg}_{3-x} \mathrm{Al}-\mathrm{LDHs}}}{\left(\mathrm{kJ} \cdot \mathrm{mol}^{-1}\right)}$ & $\frac{\Delta E_{\mathrm{Cu}_{\mathrm{x}} \mathrm{Mg}_{3-x} \mathrm{Al}-\mathrm{LDHs}}}{\left(\mathrm{kJ} \cdot \mathrm{mol}^{-1}\right)}$ \\
\hline $\mathrm{Mg}_{3} \mathrm{Al}-\mathrm{LDHs}$ (system 1) & -1580886.8 & -27446.8 \\
\hline $\mathrm{CuMg}_{2} \mathrm{Al}-\mathrm{LDHs}$ (system 2) & -1652459.4 & -26168.2 \\
\hline $\mathrm{Cu}_{2} \mathrm{MgAl}$-LDHs (system 3) & -1724034.6 & -24892.2 \\
\hline $\mathrm{Cu}_{3} \mathrm{Al}$-LDHs (system 4) & -1795373.0 & -23379.4 \\
\hline
\end{tabular}
Table 7 Binding energies of $\mathrm{Cu}_{x} \mathrm{Mg}_{3-x} \mathrm{Al}-\mathrm{LDHs}(x=0-3)$
的数目, 结果列在表 7 中.

从表 7 可看出, 从 1 号到 4 号体系的结合能绝对 值逐渐减小, 说明在 $\mathrm{Cu}_{x} \mathrm{Mg}_{3-x} \mathrm{Al}-\mathrm{LDHs}$ 体系中, 随着 原有层板上 $\mathrm{Cu}^{2+}$ 的增加, 体系的稳定性逐渐下降, 后 续逐步用 $\mathrm{Cu}^{2+}$ 取代层板上 $\mathrm{Mg}^{2+}$ 的难度增加. 同时随 着 $\mathrm{Cu}^{2+}$ 取代的增加, 虽然能带来部分姜-泰勒稳定化 能, 但 $\mathrm{Cu}^{2+}$ 本身具有的姜-泰勒畸变会很大程度地影 响层板的稳定性, 从而导致含铜水滑石体系的稳定 性降低。

\section{4 结 论}

用密度泛函理论-噟势平面波法计算了 $\mathrm{Cu}_{x} \mathrm{Mg}_{3-x} \mathrm{Al}-\mathrm{LDHs}(x=0-3)$ 体系的结构参数和电子性 质, 探讨了体系的姜-泰勒效应和结构稳定性规律.

在 $\mathrm{Cu}_{x} \mathrm{Mg}_{3-x} \mathrm{Al}-\mathrm{LDHs}(x=0-3)$ 体系中, 由于未饱 和的 $d 、 p$ 轨道共同影响着金属离子姜-泰勒畸变的 大小, 以及受周围配合物畸变的影响三方面因素, 使得金属镁的畸变角仅略小于铜的畸变角, 且随着 $\mathrm{Cu}^{2+}$ 取代 $\mathrm{Mg}^{2+}$ 的增多, 体系的平均畸变角逐渐变大, 晶胞对称性变差. 其中, 铜八面体逐渐从压扁的八 面体向稳定的拉长的八面体形式转变, 金属铝和镁 配位体分别以稳定的拉长的八面体形式稳定存在.

在 $\mathrm{Cu}_{x} \mathrm{Mg}_{3-x} \mathrm{Al}-\mathrm{LDHs}(x=0-3)$ 体系中有 $\mathrm{O}-\mathrm{H} \cdots$ $\mathrm{O}$ 型和 $\mathrm{O}-\mathrm{H} \cdots \mathrm{N}$ 型两种氢键, 并随着 $\mathrm{Cu}^{2+}$ 的增加, 两种氢键强度逐渐变弱. 另外, 随着体系中 $\mathrm{Cu}^{2+}$ 的增 加, 主客体间的静电作用力有减弱趋势. 总体上, 体 系的结合能绝对值逐渐减小, 体系稳定性下降, 故 在合成具有含铜的 LDHs 功能材料时, 应考虑层板 金属离子本身存在的姜-泰勒效应, 总体上含铜量不 宜过多.

\section{References}

(1) Kovanda, F.; Jirátová, K.; Rymeš, J.; Kolousek, D. Appl. Clay Sci. 2001, 18, 71

(2) Centi, G.; Fornasari, G.; Gobbi, C.; Livi, M.; Trifiro, F.; Vaccari, A. Catal. Today 2002, 73, 287.

(3) Busca, G.; Costantino, U.; Marmottini, F.; Montanari, T.; 
Patrono, P.; Pinzari, F.; Ramis, G. Appl. Catal. A-Gen. 2006, 310,70 .

(4) Xie, X. M.; An, X.; Yan, K.; Wu, X.; Song, J. L.; Wang, Z. Z. J. Nat. Gas Chem. 2010, 19, 77.

(5) Liu, H. B.; Jiao, Q. Z.; Zhao, Y.; Li, H. S.; Sun, C. B.; Li, X. F.; Wu, H. Y. Mater. Lett. 2010, 64, 1698.

(6) Bridier, B.; Lopez, N.; Perez-Ramirez, J. J. Catal. 2010, 269, 80.

(7) Bridier, B.; Hevia, M. A. G.; Lopez, N.; Perez-Ramirez, J. J. Catal. 2011, $278,167$.

(8) Velu, S.; Swamy, C. S. Appl. Catal. A-Gen. 1996, 145, 141.

(9) Velu, S.; Suzuki, K.; Osaki, T. Catal. Lett. 1999, 62, 159.

(10) Morpurgo, S.; Jacono, M. L.; Porta, P. J. Solid State Chem. 1996, 122, 324.

(11) Lu, R. Q.; Zhang, N. N. J. Nat. Gas Chem. 2010, 19, 179.

(12) Yan, H.; Lu, J.; Wei, M.; Ma, J.; Li, H.; He, J.; Evans, D. G.; Duan, X. J. Mol. Struct. -Theochem 2008, 866, 34.

(13) Becke, A. D. Chem. Phys. 1993, 98, 5648.

(14) Lee, C.; Yang, W.; Parr, R. G. Phys. Rev. B 1988, 37, 785.

(15) Yan, H.; Wei, M.; Ma, J.; Li, F.; Evans, D. G.; Duan, X. J. Chem. Phys. A 2009, 113, 6133.

(16) Vanderbilt, D. Phys. Rev. B 1990, 41, 7892.

(17) Xu, Q.; Ni, Z. M.; Pan, G. X.; Chen, L. T.; Liu, T. Acta Phys. -Chim. Sin. 2008, 24, 601. [胥 倩, 倪哲明, 潘国祥, 陈丽涛, 刘 婷. 物理化学学报, 2008, 24, 601.]

(18) Xu, Q.; Ni, Z. M.; Mao, J. H. J. Mol. Struct. -Theochem 2009, $915,122$.

(19) Yan, H.; Wei, W.; Ma, J.; Evans, D. G.; Duan, X. J. Phys. Chem. A 2010, 114, 7369.
(20) Ni, Z. M.; Yao, P.; Liu, X. M.; Wang, Q. Q.; Xu, Q. Chem. J. Chin. Univ. 2010, 31, 2438. [倪哲明, 姚 萍, 刘晓明, 王巧 巧, 㔻 倩. 高等学校化学学报, 2010,31, 2438.]

(21) Liu, Y. H.; Guo, Y. H.; Wu, J. Y.; Liu, L. Y.; He, J.; Chen, B. H.; Pu, M. Chem. J. Chin. Univ. 2008, 29, 1171. [刘亚辉, 郭玉华, 吴静怡, 刘灵燕, 何 静, 陈标华, 蒲 敏. 高等学校化学学报, 2008, 29, 1171.]

(22) Luo, Q. S.; Li, L.; Wang, Z. X.; Duan, X. Chin. J. Inorg. Chem. 2001, 17, 835. [罗青松, 李 蕾, 王作新, 段 雪. 无机化学 学报, 2001, 17, 835.]

(23) Cavani, F.; Trifiro, F.; Vaccari, A. Catal. Today 1991, 11, 173.

(24) Yao, P.; Ni, Z. M.; Xu, Q.; Mao, J. H.; Liu, X. M.; Wang, Q. Q. Acta. Phys. -Chim. Sin. 2010, 26, 175. [姚 萍, 倪哲明, 胥 倩, 毛江洪, 刘晓明, 王巧巧. 物理化学学报, 2010, 26, 175.]

(25) Segall, M. D.; Linda, P.; Probert, M.; Pickard, C.; Hasnip, P.; Clark, S.; Payne, M. J. Phys. -Condes. Matter 2002, 14, 2717.

(26) Ceperley, D. M.; Aider, B. J. Phys. Rev. Lett. 1980, 45, 566.

(27) Kresse, G.; Furthmiiller, J. Phys. Rev. B 1996, 54, 11169.

(28) Duan, X.; Zhang, F. Z. Assembly and Function of Intercalative Materials; Chemical Industry Press: Beijing, 2007; p 93. [段 雪, 张法智. 插层组装与功能材料. 北京: 化学工业出版 社, 2007: 93.]

(29) Pan, D. K.; Zhao, C. D.; Zheng, Z. X. The Structure of Matter; Higher Education Press: Beijing, 1989; pp 329-330. [潘道白岂, 赵成大, 郑载兴. 物质结构. 北京: 高等教育出版社, 1989: 329-330.]

(30) Mulliken, R. S. J. Chem. Phys. 1955, 23, 1833. 